\title{
Brain-Computer Interface and Classroom Teaching: Status, Bottleneck and Prospect
}

\author{
Xinyu Liu ${ }^{1,2, *}$, Yanna Ping ${ }^{1,2}$, Han $\mathrm{Li}^{1}$, Dongyun Wang ${ }^{1,2}$ \\ ${ }^{1}$ School of Intelligent Manufacturing, Huanghuai University, Zhumadian, Henan Province, China. \\ ${ }^{2}$ Henan Engineering Research Center of Intelligent Human-Machine Interaction Equipment, Huanghuai University, Zhumadian, \\ Henan Province, China.
}

\begin{abstract}
How to cite this paper: Xinyu Liu, Yanna Ping, Han Li, Dongyun Wang. (2022). Brain-Computer Interface and Classroom Teaching: Status, Bottleneck and Prospect. The Educational Review, USA, 6(2), 45-55. DOI: 10.26855/er.2022.02.002
\end{abstract}

Received: January 19, 2022

Accepted: February 12, 2022

Published: February 22, 2022

Corresponding author: Xinyu Liu, School of Intelligent Manufacturing, Huanghuai University, Zhumadian, Henan Province, China; Henan Engineering Research Center of Intelligent Human-Machine Interaction Equipment, Huanghuai University, Zhumadian, Henan Province, China.

Email: liuxinyu@huanghuai.edu.cn

\begin{abstract}
Application of brain-computer interface (BCI) in class has been reported by a large number of literatures. The current research on the BCI in classroom teaching is mainly from the perspective of researcher, but little attention has been paid to the attitude and cognition of user. Aiming at the problems of BCI in classroom teaching, a questionnaire about college students' attitudes towards the use of BCI in class was carried out. Data analysis results have shown that the prospects of BCI are relatively good in classroom teaching, but some students have insufficient understanding and have a large bias for the BCI. In addition, for the application of BCI in class, there are many bottlenecks in terms of technology maturity, cognitive biases, ethical issues, and institutional guarantees for the BCI. In-depth research is needed to find ways to crack them. We believe that the change of BCI to traditional teaching mode is limited, but combining the BCI technology with existing teaching methods will help increase student learning motivation and improve learning efficiency in class in the future.
\end{abstract}

\section{Keywords}

Brain Computer Interface, Classroom Teaching, Questionnaire, College Students

\section{Introduction}

Brain-computer interface (BCI) is a new generation of artificial intelligence technology to realize information interaction and functional integration between brain and computer by establishing a direct connection path between the brain and the external devices with high biocompatibility (Liu et al., 2020). As the most practical technology in the field of brain science, the BCI is developing rapidly with the influx of a large amount of capital. As one of the potential application fields of BCI, the education has become one of the most popular research fields at home and abroad for the BCI, and the research results have been reported in different forms of literature (Dommett et al., 2011; Purdy, 2018; Ke \& Wang, 2019).

At present, the most studied of BCI in the field of education is the neural feedback training technology for improving concentration (Heinrich, Gevensleben, \& Strehl, 2007; Hammond, 2005). Specifically, after quantifying the cognitive state of brain, it is transformed into the signals that can be perceived by the perception system through form conversion, such as sound, light, image, etc. Users adjust the intensity of the perceived signal by controlling the concentration state inside their brain, so as to improve the concentration of brain. For example, Debettencoure et al. (2015) used college students as trainers to carry out the neural feedback training, and reminded subjects of upcoming mistakes by adjusting 
the difficulty of the task itself, which significantly improved the students' performance. Nevertheless, the use of BCI in class is relatively small and controversial. At the end of 2019, the use of BCI in class by first graders of Xiangshan primary school in Zhejiang province (China) has aroused heated discussion on the Internet.

Aiming at the problem of whether the BCI is suitable for class, taking the college students who are most likely to be exposed to the BCI technology as the object, this paper makes an empirical analysis on the understanding level, application bottleneck and prospect of BCI in classroom teaching from the perspective of users by the questionnaire method. This paper includes four parts: overview of the BCI technology in classroom teaching, analysis of students' understanding level for the BCI, application bottleneck of the BCI in classroom teaching and application prospect of the BCI in classroom teaching. We systematically analyze the existing problems and future prospects of the BCI technology in classroom teaching.

\section{Overview of BCI Technology in Classroom Teaching}

The BCI consists of three parts, "brain”, “computer" and "interface”. "Brain” refers to the brain, "computer” generally refers to a computer or machine, "interface" is a device connecting the brain and the machine. The BCI focuses on the interface, which makes it possible to quantitative evaluation the changes of internal state or intention of the brain from the outside. At present, according to the implantation mode, the BCI can be divided into implantable, semi-implantable and non-implantable (Liu et al., 2020). Although the implantable and semi-implantable BCI are superior in signal quality and use effect compared with the non-implantable BCI, they need to use brain craniotomy for signal acquisition, which is expensive and high risk. Therefore, they are mainly used in animal experiments in the laboratory and in some clinical treatment of patients. Most of the common BCI in daily life are non-implantable. In the non-implantable BCI mainly collects scalp electroencephalogram (EEG), and the acquisition tools mainly include wet electrode and dry electrode. Although the signal quality of wet electrode is better than that of dry electrode, it needs the assistance of electrode jelly, which is troublesome for the use and cleaning, in signal acquisition. Therefore, it is mainly used in the laboratory at present. In the field of education, the non-implantable BCI with dry electrode is mainly used. If there is no special description in this study, the BCI mentioned refers to this form.

As a new type of intelligent interactive device, the BCI has been looking for a suitable place to use. Obviously, education is considered as one of the potential application fields. Xu, Jiang and other scholars (e.g., Xu, Chen, \& Zhang, 2018; Jiang et al., 2018) reviewed the potential impact of BCI technology in education, and looked forward to its future development prospect. Based on the research status at home and abroad, it is not difficult to find that the BCI in education, in addition to the above neural feedback training to improve concentration, another application scenario is to judge students' learning status in class, including emotion recognition, attention level measurement, etc. Verkijika et al. (2015) found that using the BCI math games in classroom teaching can effectively reduce students' anxiety. Katona et al. (2016) monitored students' attention in class through the BCI and found that the cognitive level of students participating in the project has been effectively improved. Hu et al. (2019) found that the BCI technology has shown good effectiveness in improving students' academic performance and attention, learning attitude and self-efficacy through an experimental study of 100 students for 30 days. Wang et al. (2020) used the BCI technology to classify students' learning styles and analyze students' learning attention in class. They believe that the analysis of students' attention level is of positive significance for teachers to improve teaching strategies.

Although domestic research lags behind foreign research, in the past two years, due to the pursuit of a large number of researchers, the cheap BCI equipment has developed rapidly, which has promoted domestic research to enter the implementation stage. From the above research results, it is not difficult to find that researchers generally believe that the use of BCI in the class can effectively improve learning attention and improve students' academic performance. However, these results cannot prove whether the use of BCI in class plays a "placebo" role, or whether students are curious to improve their interest in learning. In addition, there is a lack of empirical research on the use of BCI in class from the perspective of students. Based on this, this paper analyzes students' understanding level for the BCI from the perspective of students by the questionnaire method.

\section{Analysis of Students’ Understanding Level for BCI}

The questionnaire designed in this study contains 9 questions, all of which are multiple-choice questions (see Table 1). The Wechat platform is used to distribute and collect the questionnaire. A total of 1,026 questionnaires were received within 4 days. Through IP address identification, 49 duplicates were removed, and a total of 977 valid questionnaires were obtained. From the time of submitting the questionnaire, the average spend time is 54 seconds, the median is 39 seconds, the minimum value is 10 seconds and the maximum value is 1,527 seconds. It can be seen that from the 
province of the IP address submitted in the questionnaire, students from 28 provinces in China participated in the questionnaire, of which the largest number was Henan province, with 724 copies, accounting for $74.1 \%$.

\section{Table 1. Questionnaire on the use of brain computer interface (BCI) in class}

1. Sex?

$\square$ Boy $\quad \square$ Girl

2. How old are you in college?

$\square$ Freshman $\square$ Sophomore $\square$ Junior $\quad \square$ Senior

3. Do you know the BCI?

$\square$ Know and use $\quad \square$ Know but not use $\quad \square$ Never heard

4. Do you think BCI will change the lives?

$\square$ Yes $\quad \square$ No

5. Do you support the application of BCI in education?

$\square$ Support $\quad \square$ Oppose

6. Do you think BCI will help learning?

$\square$ Yes $\quad \square$ No

7. Would you like to use BCI in class?

$\square$ Yes $\quad \square$ No $\quad \square$ Confirm after understanding

8. Do you accept others using BCI in class?

$\square$ Accept $\quad \square$ Reject $\quad \square$ Don't care

9. Do you think using BCI will cause harm to your body?

$\square$ Yes, it's harmful to the brain

$\square$ Yes, it's harmful to the body

$\square$ Yes, it's harmful to the mind

$\square$ Yes, but I don't know what the harm is

$\square$ Safely

$\square$ Uncertainty

\subsection{Data Source and Statistical Analysis}

Figure 1 shows the basic situation of respondents' answers to 9 questions in the questionnaire. Among the 977 questionnaires collected, the participation of boys was higher (682), accounting for $69.8 \%$. In terms of grade distribution, it includes all grades from freshman to senior, but the number of participant's decreases from freshman to senior with a proportion of about $10 \%$. More than half of the students have never heard of the BCI, and 10.5\% have known and used it. In the item of whether the BCI will change lives, up to $80.9 \%$ chose "Yes". In the application of BCI in education, although most students choose support, the number of support is reduced by $16.3 \%$ compared with whether it will change their lives. 76.2\% of the students chose "Yes" in terms of whether it was helpful to their study. Combined with the previous question, this means that most students think that the BCI can help learning, but they lack confidence in its application in education. The results of using the BCI in class are basically consistent with expectations. $42.7 \%$ of stu- 
dents can accept the use of other students, but only $29.6 \%$ of them clearly support their own use. On the question of whether the BCI is harmful, the results are beyond our anticipation. $51.1 \%$ of the students clearly think it is harmful, only $4.1 \%$ think it is safety, and $44.8 \%$ of respondents chose "Uncertainty".
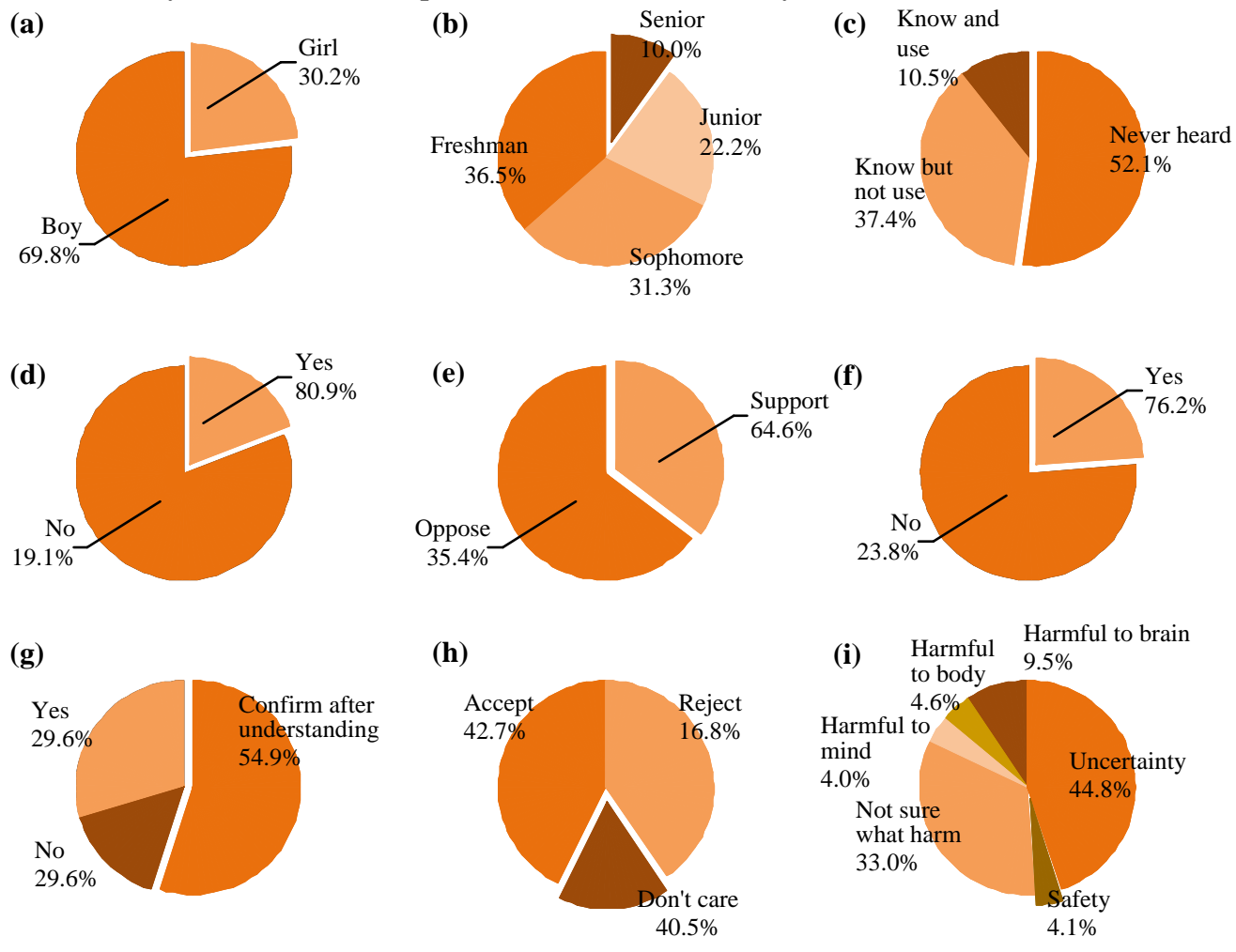

Figure 1. Overall results of the questionnaire. (a) Result of the first question. (b) Result of the second question. (c) Result of the third question. (d) Result of the fourth question. (e) Result of the fifth question. (f) Result of the sixth question. (g) Result of the seventh question. (h) Result of the eighth question. (i) Result of the ninth question.

From the results of data analysis, the BCI is still unfamiliar to the most students, they have not used it. More importantly, the most students think the BCI is harmful for their bodies. The vast majority of college students are optimistic about their prospects. Whether in the field of life or education, their support is more than $60 \%$. However, the most students are cautious about using the BCI in class. Whether they are themselves or other students, the proportion of acceptance is only $1 / 3$. This result shows that there is still a long way to go for students to really accept the application of BCI in class.

\subsection{Understanding on the Basic Function of BCI in Classroom Teaching}

In terms of understanding on the basic function of BCI in classroom teaching, the understanding of BCI by students of different genders and grades is further analyzed. The results are shown in Table 2 . We calculate the ratio of students who know and do not know the BCI. In the process of calculation, know and use as well as know but not use are classified into the same category. As can be seen from Figure 2, in terms of gender, boys and girls is basically the same for the cognitive level of BCI, but by contrast, the proportion of boys (1.07) is higher than girls (0.64) for knowing the BCI. In the grade distribution, with the growth of grade, the proportion of knowing BCI increases gradually. However, it is strange that the proportion of freshmen who know the BCI is higher than that of sophomores and juniors. This may be related to the fact that the survey time chose the second half of the semester, or it may be due to students born after the Millennium pay more attention to science and technology. The specific reason is not clear.

\subsection{Understanding on the Role of BCI in Classroom Teaching}

In terms of understanding the role of BCI in classroom teaching, this study analyzes the situation of students who know and do not know BCI in three problems: BCI changing lives, applying to education and helping to learn. The re- 
sults are shown in Figure 2. For these three problems, the support of students who know the BCI (91.9\%, 73.3\% and $84.4 \%)$ is significantly higher than that of students who do not know the BCI (70.7\%, 56.6\% and 68.8\%). This result may mean that people who know more about the BCI are more optimistic about the prospect of BCI.

Table 2. College students' understanding for the BCI

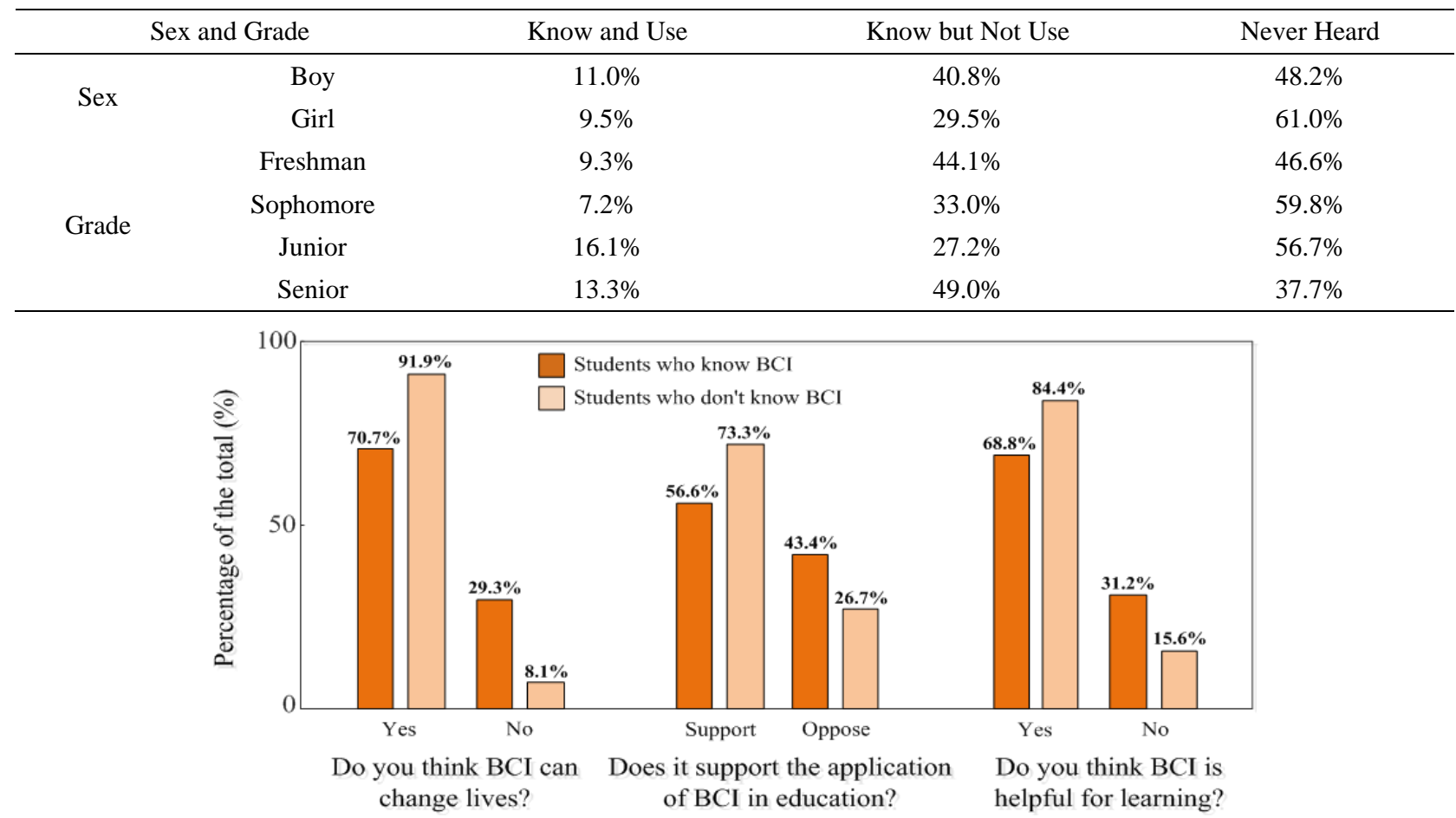

Figure 2. Comparison of students who know and do not know BCI on their understanding of the role of BCI in classroom teaching.

\subsection{Understanding on the Use of BCI in Classroom Teaching}

In the analysis of the use of BCI in classroom teaching, we also analyzed the attitudes of students who know the BCI and those who do not know the BCI in their own use and others' use respectively. The results are shown in Fig. 3. It can be seen from the figure that about half of the students who know the BCI, whether they use it by themselves or by their classmates, hold a supportive attitude, with acceptance rates of $41.5 \%$ and $54.9 \%$ respectively, and those who clearly do not support it are less than 20\%. For students who do not know the BCI, their attitude is more conservative. Most students choose a wait-and-see attitude, and $18.7 \%$ and $31.4 \%$ clearly support it. This result shows that students who know the BCI are still willing to try to use this high technology in classroom teaching.

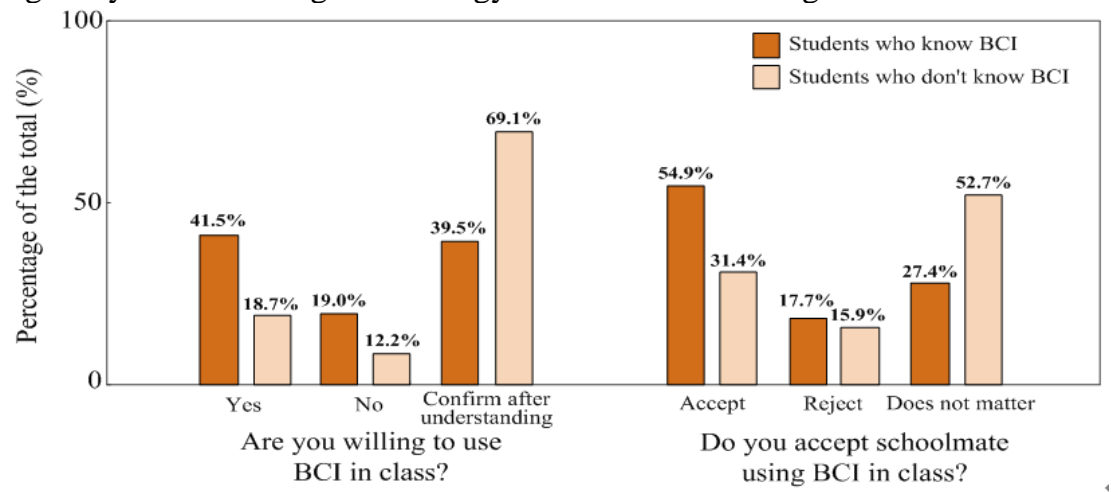

Figure 3. Comparison of students who know and do not know BCI on their understanding of the use of BCI in classroom teaching. 


\subsection{Understanding on the Harm of BCI in Classroom Teaching}

In terms of the harm of BCI in classroom teaching, we analyzed the statistics of whether students who know the BCI and those who do not know the BCI on the harm of BCI in classroom teaching. The results are shown in Figure 4. For this problem, the judgment of students who know the BCI may be more convincing, but unexpectedly, 59.8\% of students who know the BCI clearly believe that the BCI is harmful, only $7.1 \%$ think there is safety. For the students who do not know the BCI, most students (55.6\%) choose "Uncertainty" about the harm, which is also in line with the expectation. However, most students (43\%) who do not know the BCI also subconsciously believe that the BCI is harmful to the user. This result shows that most students still have prejudice against the BCI.

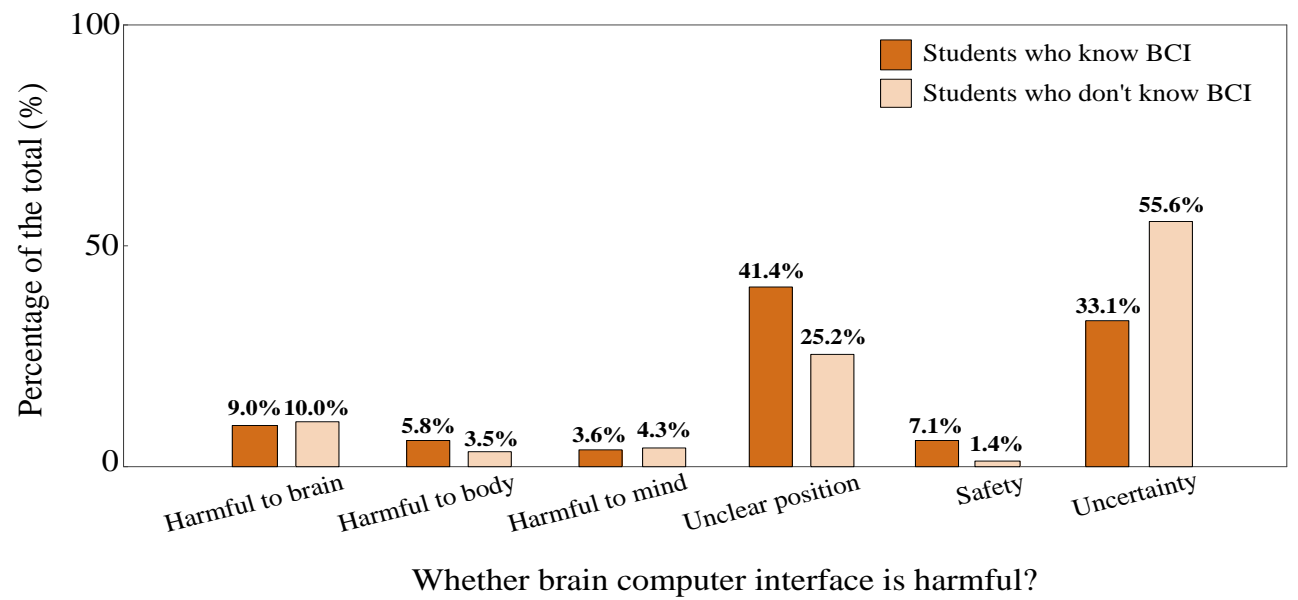

Figure 4. Comparison of students who know and do not know BCI on their understanding of the harm of BCI in classroom teaching.

\section{Application Bottleneck of BCI in Classroom Teaching}

By means of questionnaire, this study analyzes the basic attitude of college students towards the use of BCI in classroom teaching. Through the investigation of the use of BCI in university classrooms, we believe that there are still many bottleneck problems to be solved in order to use or popularize the BCI technology in classroom teaching, and whether these problems are solved or not is also directly related to whether the BCI technology can enter life and classroom from the laboratory. The application bottlenecks of the BCI technology in classroom teaching are summarized as follows.

\subsection{Technology is Immature}

The insufficient maturity of technology should be the main bottleneck affecting the application of BCI in classroom teaching. The research on the BCI can be traced back to the EEG experiment conducted by German neurologist Berger on the brain of his son Jena in 1924, and the electrical signal on human scalp was collected for the first time (Lebedev \& Nicolelis, 2006). Although the research in related fields has made great progress since then, and some rudiments of the BCI have emerged, the concept of BCI was not formally put forward until 2002 (Donoghue, 2002). The time when the BCI entered the field of education has only happened in recent years (Ren, An, \& Ling, 2019). Many studies are only limited to the experimental stage, and there is still a lot of way to go from practice.

In addition, as a cross-cutting technology, the BCI has great challenges in engineering and theory. In engineering, it is mainly reflected in: a) the signal acquisition of BCI equipment is not stable, and the signal quality needs to be improved; b) It is also inconvenient to use, and it usually needs to be used under the guidance of professionals; c) The appearance and weight need to be further optimized to reduce the psychological burden of users, so that users can wear headphones or watches without any other psychological impact; d) The use cost needs to be reasonably controlled and further improved in technology and manufacturing process; e) The development of the industry is still in its infancy, and there is a great shortage of talents in the industry, especially in the cultivation of multidisciplinary talents such as neuroscience, electronic science, information science and mechanical dynamics. In theory, the brain contains huge complexity and rich unsolved mysteries, which makes the analysis of brain mechanism very difficult. Whether it is 
"from brain to machine" or "from machine to brain", it is still in the stage of "blind men guessing an elephant". The theoretical basis is very scarce. In addition, the quantitative measurement of cognition, emotion and concentration in the brain is also immaturity.

\subsection{Insufficient Understanding}

Although the BCI has attracted more and more attention with the national attention and the entry of capital, the popularization of BCI is still slightly insufficient. From the 977 questionnaires collected, there are still $52.1 \%$ of the students who have never heard of the BCI, and only $10.5 \%$ of the students who know and use it. Moreover, the respondents of the questionnaire are the college students with higher education and most likely to be exposed to the BCI. It is not difficult to imagine that this proportion will be even more disparity for the general public. Therefore, letting more people know and accept the BCI is another bottleneck faced by BCI.

In addition, there is still a big misunderstanding about the BCI technology. In the questionnaire, only $4.1 \%$ of the students believe that the BCI is safety, and $51.1 \%$ of the students clearly believe that it is harmful, of which $9.5 \%$ think that it is harmful to the brain, $4.6 \%$ to the body, and $4.0 \%$ to the mind. What is more terrible is that many students who know BCI already believe that the BCI is harmful (59.8\%). In fact, the BCI equipment is only ordinary electronic equipment with very low electromagnetic radiation, and its harm is far lower than the common electronic equipment such as computer or mobile phone you use. People's prejudice against the unknown is understandable, but it may take more time and energy to eliminate people'+s prejudice against the BCI.

\subsection{Ethical Issues}

Although the ethical issues of BCI have not received extensive attention, with the gradual maturity of the BCI technology and the use of more and more fields, ethical issues will become a major bottleneck of the BCI technology (Ning, Cao, \& Zhang, 2018), especially in classroom teaching. In which, we believe that the ethical problems of BCI mainly exist in the following aspects:

a) Personal privacy issues. The BCI takes the brain as the research object, and can read the internal privacy information such as the brain's intention, emotion, preference, concentration and health status. In the future, with the maturity of technology, there will only be more and more readable information and most of this information are very secret. If the collected data is not well protected, it will greatly infringe on the user's privacy.

b) The right to informed consent. The right to informed consent is one of everyone's basic rights. As a high and new technology, people do not fully understand its function and role, and most information collectors do not inform users of the detailed data collection purpose for various purposes, especially for students in vulnerable groups. They may be minors in most cases, and their awareness of self-protection is still very weak. Therefore, the user's right to informed consent may be greatly violated.

c) Education equity. China's education policy is to maintain educational equity. However, if the BCI may help greatly improve students' cognitive ability with the progress of technology, it will significantly improve the teaching quality in the classroom. However, limited by external factors such as technology, politics and development level, it is very likely that only a few students will have the conditions and capital to promote "evolution". Thus, through less pay, they can become "top students" in a sense. This leap of "carp leaping to the dragon's gate" obtained by relying on modern high-tech technology will break the existing educational equity policy and trigger greater ethical concerns.

\subsection{Imperfect System Guarantee}

The BCI has a market potential of hundreds of billions of dollars in the field of Education (Chen \& Wang, 2019), and its huge market value will also promote the influx of greater capital. In the process of BCI marketization, how to protect the rights of students as the weak party has become an urgent problem. At present, in the classroom teaching, even in the whole field of education, the relevant laws and regulations on the BCI are almost blank. Moreover, the BCI technology still has many ethical problems, such as students' right to privacy, informed consent and educational equity, which need strong laws and regulations.

At present, schools in developed coastal areas have begun to try the BCI technology in class. It can be predicted that in the near future, with the gradual maturity of BCI technology, more and more "crab eaters" will let the BCI enter the class. Therefore, it is urgent to establish perfect laws and regulations to protect the rights and interests of users. Although the research results related to the BCI are of great significance to improve the quality of classroom teaching, perfect system guarantee must be accompanied. 


\section{Application Prospect of BCI in Classroom Teaching}

With the gradual deepening of people's understanding of the external world and the laws of nature, the modern science and technology have developed vigorously. But in today's society, it is rare that science can effectively solve the problems of classroom teaching. As the closest to classroom teaching, brain science has been paid more and more attention by scholars. Although there are still many bottlenecks, as the most mature brain science technology, the application prospect of BCI is still very promising. At present, the research on the BCI in classroom teaching mainly focuses on non-mainstream fields, such as judging students' concentration, identifying students' cognitive and emotional states, and eliminating students' anxiety (Wei, 2020), so as to provide reference and basis for teachers' teaching strategies or classroom teaching design. In addition, we believe that the BCI will have broad application prospects in the following fields in classroom teaching.

\subsection{Evaluation of Classroom Teaching Quality}

Classroom teaching quality evaluation has always been criticized by teachers at all stages and grades, because the results of teaching quality evaluation will directly affect the promotion of each teacher's professional title, year-end bonus and the acquisition of teaching related honors. Although different schools have developed a variety of teaching evaluation methods, the results are not satisfactory. Because these teaching evaluation methods are obtained quantitatively based on students' scoring, there is a lot of room for artificial manipulation, and many students do not take them seriously. At present, there are not many solutions. However, the BCI may provide a new solution for classroom teaching quality evaluation in the future.

When using the BCI to evaluate the teaching quality, on the one hand, the BCI can be used to record the concentration time and learning status of each student in each lesson, calculate a score for each teacher in it, and let the teaching quality evaluation run through each lesson, which will greatly reduce the impact of subjective factors on the evaluation results. On the other hand, we can also monitor the brain activity of each student in the process of teaching evaluation, and then eliminate the teaching evaluation results of those students who do not take teaching evaluation seriously, so as to improve the credibility of teaching quality evaluation. With the maturity and in-depth research of the BCI technology, better solutions may appear in this field in the future to solve the teaching evaluation problems closely related to each teacher.

\subsection{Performance Measurement of Teaching Methods}

In classroom teaching, there are many teaching methods. However, whether it is traditional questioning method, situational method, modern flipped classroom, CDIO engineering education, etc., there is a lack of an effective tool to evaluate the quality of these methods. Although it may not have much practical significance to evaluate the advantages and disadvantages of a teaching method in the actual classroom teaching, it may be very necessary to use a quantitative method to judge the advantages and disadvantages of a teaching method in the teaching competition, which greatly weakens the impact of the judges' personal subjective factors on the competition results and improves the fairness and impartiality of the competition.

The purpose of using different teaching methods is to better enable students to obtain knowledge and improve their interest in learning, so as to improve the quality of teaching. When using the BCI to measure the effect of teaching methods, the changes of brain cognitive state or function of each student before and after class can be detected in the competition, and this change can be used to quantitatively evaluate whether the teaching methods can effectively improve the learning effect of students. In this way, it can not only assist the judges to judge the teaching methods, but also directly participate in the teaching effect evaluation, so as to reduce the influence of human factors. Although it is not mature to use the BCI to judge the teaching effect, with the gradual improvement of the BCI technology, it will have a good prospect in the measurement of teaching method effect.

\subsection{Discrimination of Learning Style}

In addition to the above two applications, the discrimination of learning style is also one of the hot directions in research of BCI for classroom teaching. In this direction, most studies mainly focus on measuring students' emotional state by using the BCI technology, so as to help teachers improve students' learning enthusiasm. For example, Wang (2020) combined the BCI with mobile terminal to identify students' learning style, and matched teachers' teaching style with learning style to improve teaching strategy. Mehmood et al. (2017) put forward a computer-aided teaching method. They identify the current emotional state of the brain through the BCI technology to help teachers in special schools 
improve students' learning ability.

In addition, in division of Arts and Sciences, the BCI technology can also be used to identify students' Arts or Sciences preferences. At present, most provinces will divide Arts and Sciences in high school. In division of Arts and Sciences, most of them are classified based on students' learning expertise, which is lack of theoretical basis. And after the division is completed, students will be trained according to different modes. Once they make a wrong choice, it will affect students' career in the future. Therefore, it is very important to improve the accuracy of division. In division of Arts and Sciences, we can better judge whether students are suitable for Arts learning or Science learning through students' EEG signals, then feed it back to the students to help them make the right choice between Arts and Science.

\subsection{Monitoring of Students’ Learning Status}

In learning state monitoring, the measurement of attention or concentration level is a main research direction of the BCI for classroom teaching, and the theoretical basis is relatively mature. For example, the average attention measurement system based on the BCI technology designed by Katona et al. (2016) is of great help to improve the attention level during class through the feedback of students' use effect. Ke et al. (2019) collected the changes of students' attention during reading and writing through the BCI technology, and found that through the changes of attention, different students' cognitive styles can be distinguished, such as visual, auditory, reading and writing, operational, etc. By teaching students with different cognitive styles according to their aptitude, the learning effect can be improved.

In addition to attention and emotion monitoring, the BCI also has potential application directions in learning state monitoring, such as fatigue state monitoring. At present, the monitoring of fatigue status is mainly applied in the field of transportation, such as judging whether the driver is driving fatigue. There is no relevant research in the classroom teaching. There are at least two advantages to detect the fatigue state in class: first, it can timely find the students in poor class state, and can timely remind and find the problems of students as soon as possible; second, we can monitor the overall learning state of students. If we find that students are tired as a whole, we can appropriately change the teaching mode and improve students' mental state. Of course, there may be other forms or contents in class mental state monitoring, which need further research and thinking.

\subsection{Auxiliary Tools for Education or Entertainment}

Education and entertainment is also a major application direction of the BCI. At present, the proliferation of smart phones in society has greatly improved the convenience of students' communication, but it also shows many disadvantages. For example, some students have poor self-control and use their mobile phones to indulge in social software chat, mobile games, Internet surfing and other activities, which seriously affects their learning state and mental laxity in the classroom teaching. At present, researchers have developed many brain-controlled games by using the BCI technology, such as brain-controlled spider, brain-controlled racing car, brain-controlled game console, etc., which can train students' brain concentration in their extracurricular pastime and improve their cognitive level. In addition, other functions can also be added to the BCI, such as communication, timing, assisted sleep, health monitoring and other functions, so that the BCI can be truly applied to life and serve students.

In the field of supplementary education and entertainment, the BCI has another major application direction, which is to assist special education. For example, at present, the brain-controlled typing technology is relatively mature. Using ideas can achieve the fastest typing of 69 Chinese characters per minute, which has exceeded the speed of handwritten Chinese characters, which is a blessing for many disabled students. In addition, there are brain-controlled Book flipping and brain-controlled wheelchairs, which will undoubtedly broaden the current education model and improve the conditions of education. It can be predicted that in addition to the current brain-controlled external equipment to improve the self-care ability of disabled students. With the maturity of BCI technology, it will be very useful in the field of special education.

\section{Conclusion}

From medical rehabilitation to smart home, from games and entertainment to educational technology, the BCI affects several high-tech fields with intelligent interaction as the core. Especially in the field of educational science and technology, the BCI technology is considered to have great potential in both the classroom teaching and the educational assistance. Based on the questionnaire survey, this paper analyzes the students' understanding about BCI, and discusses the application bottleneck and application prospect of the BCI. The specific conclusions are as follows: a) although the BCI technology has been studied in the classroom teaching, and most students are also very optimistic about its prospect, However, students do not understand BCI, their cognition of BCI is also one-sided, their acceptance is not very 
high, and the task of science popularization has a long way to go; b) There are still many bottlenecks in the application of BCI in the classroom teaching, mainly in four aspects: immature technology, cognitive bias, many ethical problems and imperfect system guarantee, which need to be studied; c) There are many fields worth developing in the application prospect, such as the evaluation of teaching quality, the measurement of the effect of teaching methods, the discrimination of learning styles, the monitoring of mental state and the assistance of education and entertainment, which will be of great use in these non-mainstream fields.

Although the BCI technology has been widely used and studied in the classroom teaching, even in the field of educational science and technology, it still follows the non-mainstream line. The theoretical basis is not perfect and the role is not obvious. In addition, in education, the BCI mainly focuses on the human, involves too many ethical issues, and greatly limits its research and development. More importantly, students are a special group. Without sufficient grasp and evidence, it is difficult to persuade education managers to support this research, which also hinders its development in the classroom teaching. Therefore, on the whole, in education, the BCI is still in the laboratory stage, and it is too early to enter the campus or classroom. At present, the use of BCI in the classroom teaching is only a gimmick. For example, the BCI incident of Xiangshan primary school of Zhejiang province (China) in 2019 has attracted more criticism, which is worth pondering.

In the field of education or classroom teaching, the research on the BCI needs to be further strengthened. We believe that the research direction should mainly focus on the following directions: a) deeply carry out the research on brain neural mechanism related to the classroom teaching such as learning, cognition and attention, so as to provide a theoretical basis for the application of BCI in the classroom teaching; b) Improve the acquisition and processing technology of scalp EEG signals, strengthen the update of software algorithms, and provide technical support for the application of $\mathrm{BCI}$ in the classroom teaching; c) The manufacturing process and wearing mode of BCI are further improved to make the wearing and use of BCI more convenient; d) Strengthen the publicity and popularization of BCI technology, develop more brain control equipment, and make the BCI really enter people's life, eliminate public misunderstanding and suspicion of the BCI, and gradually accept it; e) Improving relevant laws, regulations and systems, effectively protecting users' privacy and rights, and putting brain computer interfaces into the cage of the system is not only the protection of BCI users, but also the supervision of their developers. It takes time for science and technology to be accepted and understood by the public. We should give more support and encouragement to scientific research workers, so that scientific research achievements can truly be indomitable and serve the people.

\section{Acknowledgements}

This research was funded by Young Teacher Foundation of Huanghuai University; "Creating youth" China Youth Innovation and Entrepreneurship Project; Collaborative education project of industry university cooperation of the Ministry of Education (202002131003); Key Scientific Research Projects of Colleges and Universities in Henan Province (21A413004).

\section{References}

Chen, L. N. and Wang, D. Z. (2019). Research progress in responsible innovation of brain-computer interface. Journal of Engineering Studies, 11, 390-399.

Debettencoure, M. T., Cohen, J. D., Lee, R. F., Norman, K. A., and Turk-Browne, N. B. (2015). Closed-loop training of attention with real-time brain imaging. Nature Neuroscience, 18, 470-475.

Dommett, E. J., Devonshire, I. M., Plateau, C. R., Westwell, M. S., and Greefield, S. A. (2011). From scientific theory to classroom practice. The Neuroscientist, 17, 382-388.

Donoghue, J. P. (2002). Connecting cortex to machines: recent advances in brain interfaces. Nature Neuroscience, 5, $1085-1088$.

Hammond, D. C. (2005). Neurofeedback with anxiety and affective disorders. Child and Adolescent Psychiatric Clinics of North America, 2005, 14, 105-123.

Heinrich, H., Gevensleben, H., and Strehl, U. (2007). Annotation: neurofeedback-train your brain to train behavior. Journal of Child Psychology and Psychiatry, 48, 3-16.

Hu, H., Li, Y. X., Cao, Y. F., Zhao, Q. H., and Lang, Q. E. (2019). The path and experimental research of brain-computer interaction promoting deep learning: attention intervention analysis in the online learning system. Journal of Distance Education, 4, 54-63.

Jiang, L., Zhang, H., Zhang, L., Wu, C., Sun, Q. C., and Li, H. B. (2018). Mapping knowledge domains analysis on evolution of BCI 
and potential application in educational field: based on journals of SCI and SSCI from 1985 to 2018. Journal of Distance Education, 4, 27-38.

Katona, J. and Kovari, A. (2016). A brain-computer interface project applied in computer engineering. IEEE Transactions on Education, 59, 319-326.

Ke, Q. C. and Wang, P. L. (2019). Research progress on the application of brain-computer interface technology in education. China Educational Technology, 10, 14-22.

Lebedev, M. A. and Nicolelis, M. A. L. (2006). Brain-machine interfaces: past, present and future. Trends in Neurosciences, 29(9), 536-546.

Li, X. Y., Chen, F., Jia, Y. H., and Liu, X. Y. (2020). Signal detection, processing and challenges of non-invasive brain-computer interface technology. Lecture Notes in Electrical Engineering, 586, 60-67.

Mehmood, R. M. and Lee, H. J. (2017). Towards building a computer aided education system for special students using wearable sensor technologies. Sensors, 17, 1-22.

Ning, X. L., Cao, Y. F., and Zhang, Y. (2018). Analysis of ethical issues in the application of brain-computer interface technology. Medicine \& Philosophy, 39, 35-38.

Purdy, N. (2018). Neuroscience and education: how best to filter out the neurononsense from our classrooms. Irish Educational Studies, 27, 197-208.

Ren, Y., An, T., and Ling, R. (2019). Brain-computer interface technology education application: status, trends and challenges. Modern Distance Education, 2, 71-78.

Verkijika, S. F. and De-Wet, L. (2015). Using a brain-computer interface (BCI) in reducing math anxiety: evidence from South Africa. Computers and Education, 81, 113-122.

Wang, P. L., Ke, Q. C., and Zhang, J. Q. (2020). Research on application of brain-computer interface in smart classroom. Open Education Research, 26, 72-81.

Wei, N. (2020). Brain computer interface: a non-mainstream line of artificial intelligence education application. China Information Technology Education, 1, 16.

Xu, Z. G., Chen, Q. H., and Zhang, G. W. (2018). A new generation of human-computer interaction: the status, types and educational application of natural user interface: also on the preliminary outlook of brain-computer interface technology. Journal of Distance Education, 4, 39-48. 